\title{
Demo: Multi-Scale Gestural Interaction for Augmented Reality
}

\author{
Barrett Ens \\ University of South Australiay \\ Adelaide, Australia \\ barrett.ens@unisa.edu.au
}

\author{
Aaron Quigley \\ University of St. Andrews \\ St. Andrews, Scotland \\ aquigley@st-andrews.ac.uk
}

\author{
Hui-Shyong Yeo \\ University of St. Andrews \\ St. Andrews, Scotland \\ hsy@st-andrews.ac.uk
}

\author{
Pourang Irani \\ University of Manitoba \\ Winnipeg, Canada \\ pourang.irani@cs.umanitoba.ca
}

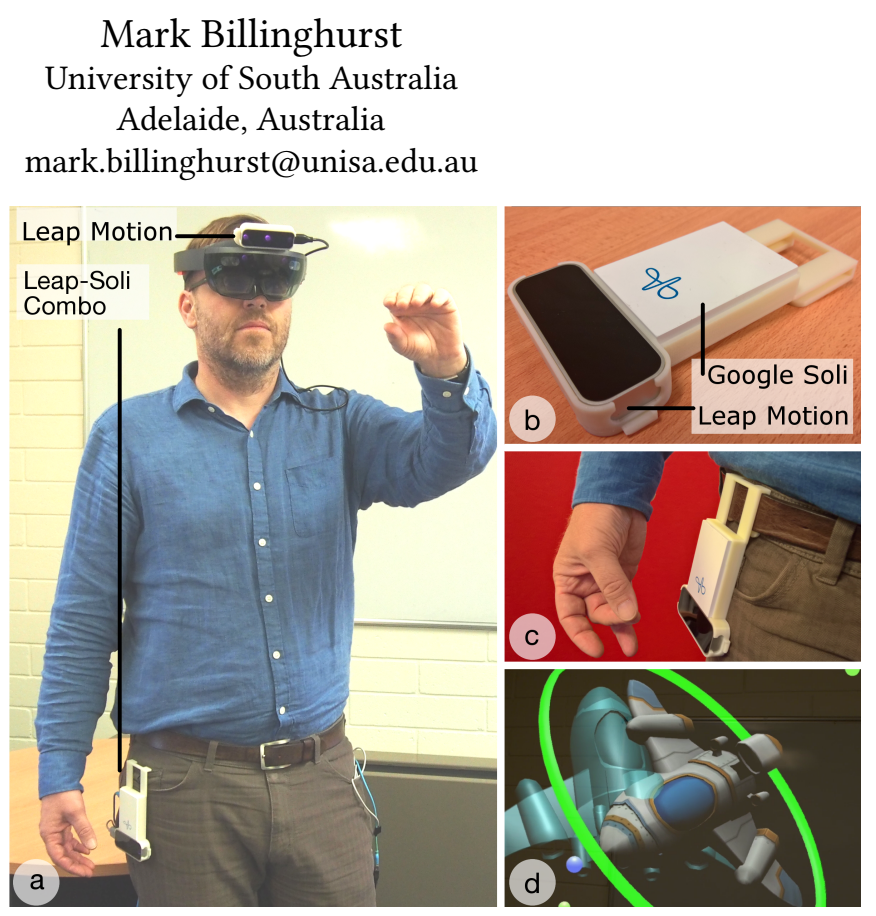

Figure 1: A head-mounted Leap sensor (a) along with a Leap+Soli configuration (b) worn on the user's belt (c). This configuration allows both direct manipulation and precise control in applications such as a docking task (d).

\section{MULTI-SCALE GESTURES}

Gestures, including gesticulation, language like, pantomime or emblematic movements [Wu and Huang 1999], are a natural part of human communication. Interaction designers have long sought sensing technologies that allow hand gestures to be sensed and interpreted, eliminating altogether the need for mechanical input devices. Researchers have incorporated pointing, grasping and waving gestures in numerous contexts. We present a prototype AR interface (fig. 1) that combines interaction on multiple scales, using

(c) ACM, 2014. This is the author's version of the work. It is posted here by permission of ACM for your personal use. Not for redistribution. The definitive version will be published in SA '17 Symposium on Mobile Graphics \& Interactive Applications

November 27-30, 2017, Bangkok, Thailand

(c) 2017 Copyright held by the owner/author(s).

multiple wearable sensors. A Leap Motion [Leap Motion 2017] sensor mounted on a HoloLens [Microsoft 2017] allows macro-scale direct manipulation of virtual objects. A belt configuration, which includes a second Leap Motion sensor combined with a Google Soli [Google 2017; Lien et al. 2016], allows fine-scale object manipulation using microgestures, when the arm is in a relaxed, low-fatigue posture. This work builds on previous research [Ens et al. 2016; Liu et al. 2015] that allows gesture input with a relaxed arm posture. Whereas the system by Ens et al. [Ens et al. 2016] relies on a ring device, our belt-worn sensor configuration leaves the hand unencumbered and allows richer interaction. We will present several applications that provide precise, low fatigue interaction with smooth transitions between macro- and micro-gesture scales. For instance, a docking task uses six virtual sliders mapped onto the tips and sides of three different fingers, to precisely control six degrees of freedom of a virtual object (fig. 1d). 


\section{REFERENCES}

Barrett Ens, Ahmad Byagowi, Teng Han, Juan David Hincapié-Ramos, and Pourang Irani. 2016. Combining ring input with hand tracking for precise, natural interaction with spatial analytic interfaces. In Proceedings of the 2016 Symposium on Spatia User Interaction - SUI '16. ACM Press, New York, New York, USA, 99-102. https: //doi.org/10.1145/2983310.2985757

Google. 2017. Project Soli. (2017). https://atap.google.com/soli/ (Date last accessed 15-September-2017)

Leap Motion. 2017. Leap Motion. (2017). https://www.leapmotion.com/ (Date last accessed 15-September-2017).

Jaime Lien, Nicholas Gillian, M. Emre Karagozler, Patrick Amihood, Carsten Schwesig, Erik Olson, Hakim Raja, and Ivan Poupyrev. 2016. Soli: Ubiquitous gesture sensing with millimeter wave radar. ACM Transactions on Graphics 35, 4 (July 2016), 1-19. https://doi.org/10.1145/2897824.2925953

Mingyu Liu, Mathieu Nancel, and Daniel Vogel. 2015. Gunslinger: Subtle arms-down mid-air interaction. In Proceedings of the 28th Annual ACM Symposium on User Interface Software \& Technology - UIST '15. ACM Press, New York, New York, USA 63-71. https://doi.org/10.1145/2807442.2807489

Microsoft. 2017. The leader in Mixed Reality Technology | HoloLens. (2017). https //www.microsoft.com/en-us/hololens

Y Wu and TS Huang. 1999. Vision-based gesture recognition: A review. Gesture Workshop (1999). http://link.springer.com/content/pdf/10.1007/3-540-46616-9.pdf 